\title{
Clopidogrel Hyper-Response and Bleeding Risk in Neurointerventional Procedures
}

\author{
C. Goh, L. Churilov, P. Mitchell, R. Dowling, and B. Yan
}

\begin{abstract}
BACKGROUND AND PURPOSE: Antiplatelet therapy is associated with decreased ischemic events after neurointerventional procedures. Antiplatelet resistance negates the protective effects of antiplatelet medication, leading to a higher incidence of ischemic events. A possible link between antiplatelet hyper-response and increased hemorrhagic complications has been inadequately investigated. We aimed to examine the correlation between antiplatelet hyper-response and the risk of hemorrhagic complications.
\end{abstract}

MATERIALS AND METHODS: Patients who were treated with antiplatelet medications and underwent neurointerventional procedures were prospectively recruited. We collected the following data: demographics, vascular risk factors, antiplatelet and anticoagulation treatment, antiplatelet responsiveness, coagulation profile, and hemorrhagic complications. P2Y12 receptor-mediated platelet inhibition was tested by using the VerifyNow assay device. The primary end points were postprocedural major and minor hemorrhagic complications. Receiver operator characteristic analysis was used to evaluate the percentage of platelet inhibition as a diagnostic tool for bleeding events. The association between hemorrhage and percentage of platelet inhibition was investigated by using logistic regression modeling.

RESULTS: Forty-seven patients were enrolled. The mean age was $56 \pm 12$ years, and $28 \%$ were men. Ten patients (21.3\%) developed hemorrhagic complications. Clopidogrel response was higher in patients with a major bleeding complication compared with those with minor or no bleeding (median, $94 \%$ versus $24 \%$ platelet inhibition; $P=.0084)$. Of the 7 patients $(14.9 \%)$ defined as hyper-responders with $\geq 72 \%$ platelet inhibition, $42.8 \%$ had a major bleeding complication.

CONCLUSIONS: Hyper-response to clopidogrel is associated with increased risk of hemorrhagic complications. Larger studies are urgently needed to validate a clinically useful threshold to define clopidogrel hyper-response and to examine the clinical effects of antiplatelet dosage adjustment.

ABBREVIATIONS: $\mathrm{ACT}=$ activated clotting time; aPTT = activated partial thromboplastin time; $\mathrm{Cl}=$ confidence interval; IQR $=$ interquartile range; $\mathrm{ROC}=$ receiver operating characteristic

D ual antiplatelet therapy with aspirin and clopidogrel is associated with reduced risk of thromboembolic complications of endovascular neurointerventions ${ }^{1}$ and in percutaneous coronary intervention. ${ }^{2}$ However, it is well-recognized that there is a high level of interindividual variability in the antiplatelet response to clopidogrel. ${ }^{3-5}$ Hypo-response to clopidogrel has been shown to be associated with a higher incidence of adverse outcomes, including 3.4-fold increase in cardiovascular mortality, 3-fold in-

Received June 18, 2012; accepted after revision September 19.

From the Department of Radiology (C.G., R.D.), Neurointervention Service (R.D.,

P.M., B.Y.), and Comprehensive Stroke Centre (B.Y.), Royal Melbourne Hospital,

Melbourne, Australia; Department of Mathematics and Statistics (L.C.), University

of Melbourne, Melbourne, Australia; and National Stroke Research Unit (L.C.), Florey Neuroscience Institute, Melbourne, Australia.

Please address correspondence to Prof. Bernard Yan, Division of Neurosciences, Royal Melbourne Hospital, Grattan St, Parkville, Victoria 3050, Australia; e-mail address: bernard.yan@mh.org.au

http://dx.doi.org/10.3174/ajnr.A3418 crease in nonfatal myocardial infarction, and 4-fold increase in stent thrombosis. ${ }^{3,6-9}$

Genetic polymorphism of the CYP2C19 allele, crucial to metabolism of clopidogrel into its active metabolite, has been implicated as a mechanism of both hyper- and hypo-response. The interest in this area is demonstrable, with the institution of a boxed warning by the US Food and Drug Administration regarding the risk of adverse outcomes in carriers of the implicated variants. ${ }^{10}$

Of note, there exists a subset of patients with hyper-response to clopidogrel who may be at increased risk of hemorrhagic complications. ${ }^{11,12}$ There are few studies that quantify the incidence of hemorrhage in patients with a high level of response to the antiplatelet effects of clopidogrel, and it remains unclear whether testing for response to antiplatelet therapy and adjustment of antiplatelet therapy will have an impact on bleeding risk. In addition, previous results arose from studies of coronary intervention. ${ }^{11,13}$ 


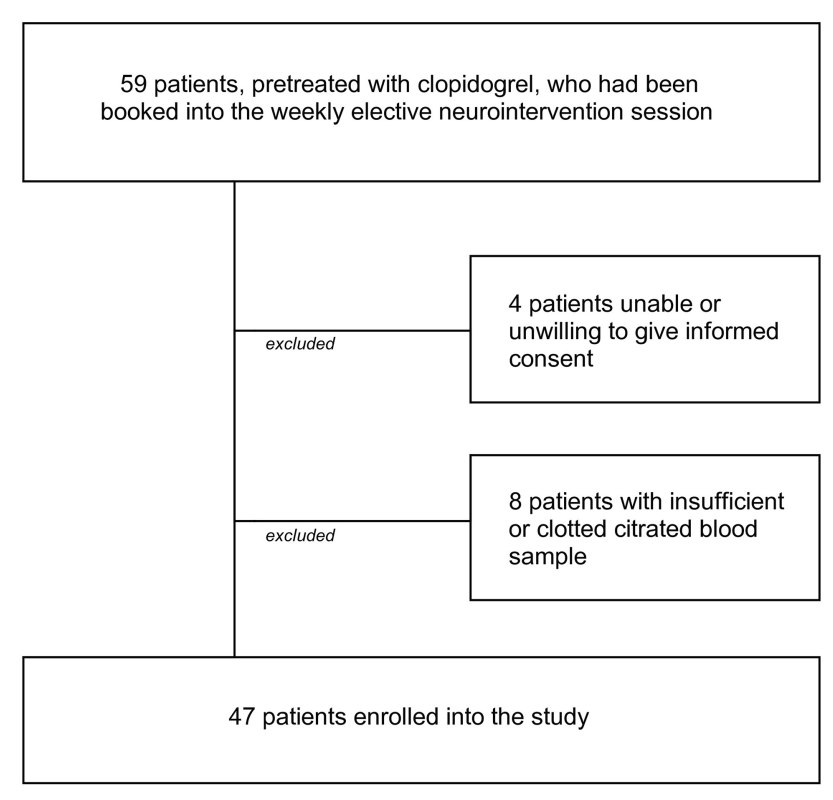

FIG 1. Flow chart illustration of patient enrollment.

Patients undergoing endovascular neurointervention may differ from the percutaneous coronary intervention population in terms of demographics and cardiovascular risk factors, particularly those being treated for cerebral aneurysm. This difference has the potential to alter the balance of risk between ischemic complications of the procedure and hemorrhagic complications of antiplatelet treatment.

This study aimed to describe the incidence of hyper-response to clopidogrel in the population of patients undergoing neurointervention and to investigate the association between clopidogrel hyper-response and the risk of hemorrhagic complications.

\section{MATERIALS AND METHODS}

Patients were recruited from those undergoing endovascular neurointervention during the weekly elective session at the Royal Melbourne Hospital between May 2010 and May 2011 (Fig 1). Patients were excluded if they refused consent, were younger than 18 years of age, or had a history of an acute event (transient ischemic attack, ischemic infarct, or acute intracranial hemorrhage) within the 24 hours before the procedure. Demographic and clinical data collected included age, sex, and cardiovascular risk factors (hypertension, dyslipidemia, diabetes mellitus, smoking history, and history of previous cerebrovascular ischemic events). The study protocol received ethics committee approval.

The standard antiplatelet regimen at our institution is dual therapy with clopidogrel, $75 \mathrm{mg}$, and aspirin, $100 \mathrm{mg}$, for the 3 days before an endovascular neurointerventional procedure.

All procedures were performed by 1 of 3 experienced neurointerventionalists. Arterial access was via a $6 \mathrm{~F}$ femoral sheath, with hemostasis obtained postprocedure by use of an Angio-Seal device (St. Jude Medical, Minnetonka, Minnesota). Systemic anticoagulation with intravenous heparin during the procedure was titrated to an ACT twice normal. The postprocedural unfractionated heparin infusion rate was adjusted on the basis of aPTT.

Whole blood was obtained for testing at the time of the initial femoral puncture; following discard of the first $5 \mathrm{~mL}$, citrated
Vacette tubes (VWR, Arlington Heights, Illinois) were filled and inverted 5 times with blood analyzed by using the VerifyNow assay device (Accumetrics, San Diego, California).

The VerifyNow assay is a point-of-care device using a 2-channel disposable cartridge, with platelet aggregation measured by increasing light absorbance through the sample. The first chamber contains a strong agonist for platelet activation independent of aspirin or clopidogrel therapy, providing a baseline platelet function measurement (BASE). The second chamber gives a measurement expressed as $\mathrm{P} 2 \mathrm{Y} 12$ reaction units (PRU), representing platelet activation by the adenosine diphosphate-P2Y12 pathway. Percentage of inhibition is calculated from the formula [1 (PRU/BASE)]/100. The VerifyNow assay has been shown to correlate well with the current criterion standard light transmittance aggregometry and with other point-of-care assay devices, ${ }^{14-16}$ and it has been validated in a head-to-head comparison of assay methods based on outcome measures. ${ }^{17}$

Outcomes were measured by clinical assessment during the hospital admission and at the 6-week review appointment. The primary end point was Thrombolysis in Myocardial Infarction major or minor periprocedural bleeding events. The need for investigation with Doppler sonography or CT or MR imaging of the brain was guided by clinical assessment.

Statistical analysis was performed by using the commercial statistical software STATA, Version 11IC (StataCorp, College Station, Texas). Platelet inhibition is reported as median IQR due to non-normal distribution as assessed by the Shapiro-Wilk test. The Wilcoxon-Mann-Whitney test was used to compare the percentage of platelet inhibition by clopidogrel between bleeding and nonbleeding groups with the corresponding effect size estimated by using median regression. The receiver operator characteristic analysis curve was used to evaluate the percentage of platelet inhibition measured by the VerifyNow assay device as a diagnostic tool for bleeding events. Association between the presence of periprocedural bleeding and the percentage of platelet inhibition was investigated by using logistic regression modeling (both unadjusted and adjusted for mean aPTT and intraprocedural ACT). The statistical significance threshold for all the tests performed was set as $P=.05$.

\section{RESULTS \\ Study Population}

Forty-seven patients were enrolled in the study, 34 women and 13 men, with a mean age of $56 \pm 12$ years (range, $30-78$ years). There was a history of hypertension in $46.8 \%$, dyslipidemia in $25.5 \%$, and diabetes mellitus in $4.3 \%$ of patients, and $29.8 \%$ were current smokers. Previous TIA or ischemic stroke was documented in only $0.9 \%$.

\section{Procedure}

All endovascular procedures were performed by 1 of 3 neurointerventionists. All except 1 in the study population received treatment for an intracranial aneurysm, by coil embolization alone (23.4\%), with balloon remodelling $(21.3 \%)$ or stent assistance $(19.1 \%)$ or by use of a flow-diverting stent $(34 \%)$. The other patient underwent stent placement for a vertebral artery stenosis. All except 9 patients received ongoing systemic anticoagulation with 


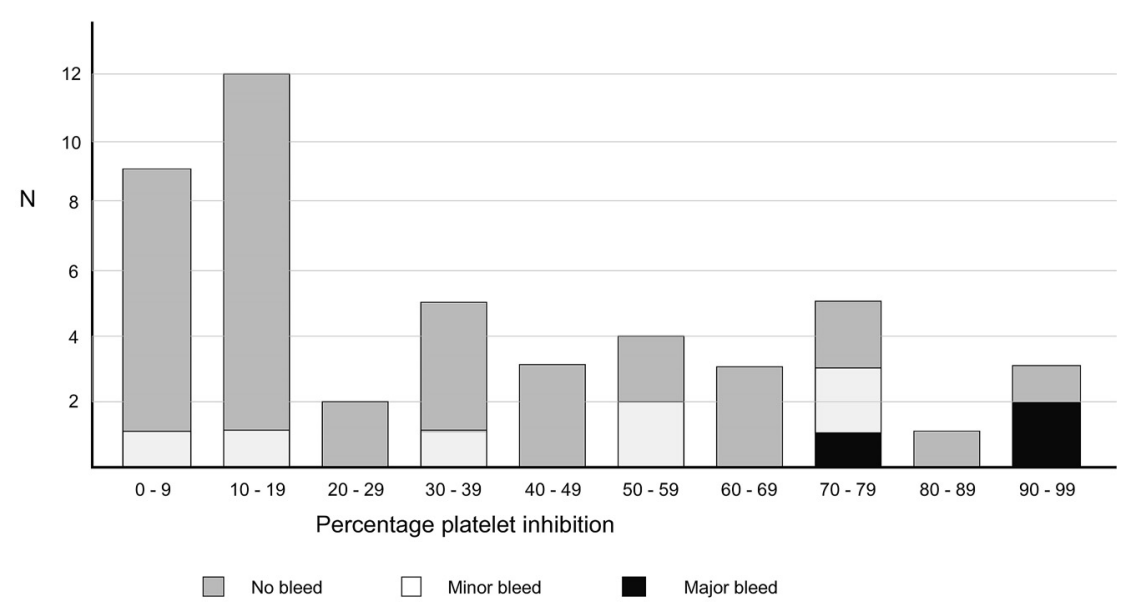

FIG 2. The distribution among the study population of major and minor bleeding events and the level of platelet inhibition achieved.

intravenous heparin following the procedure: 21 patients for up to 24 hours and 17 patients for 48 hours. One patient who had a Factor V Leiden deficiency received 10 hours of intravenous unfractionated heparin postprocedure and then low-molecularweight heparin until therapeutic anticoagulation with warfarin had been achieved.

Treatment of 3 patients deviated from the standard antiplatelet regimen. Two received a single loading dose of $450 \mathrm{mg}$ of clopidogrel at 3 hours or at 5 hours before the procedure. The other had a history of Factor V Leiden deficiency and had been receiving subcutaneous low-molecular-weight heparin due to cessation of his usual long-term anticoagulation with warfarin. The decision was made to give him 3 days of clopidogrel loading at $75 \mathrm{mg}$ daily, rather than dual antiplatelet therapy.

\section{Clopidogrel Response and Bleeding Events}

Response to the antiplatelet effects of clopidogrel varied widely, with the percentage of platelet inhibition ranging between $0 \%$ and 99\% (median, 30\%; IQR, 12\%-61\%). Figures 2 and 3 illustrate the distribution of platelet inhibition and bleeding events among the study population.

Eleven patients had a bleeding event during the procedure or in the 24 hours postprocedure. Thrombolysis in Myocardial Infarction major bleeding events occurred in 3 patients (6.4\%). Two of these had documented periprocedural intracranial hemorrhages. A 5-mm left temporal intracerebral bleed occurred in a patient with 99\% platelet inhibition and PRU 5, whose right posterior communicating artery aneurysm was treated by placement of a flow-diverting stent. MR imaging of the brain showed no evidence of an underlying cavernoma, mass, or infarct. A tiny subarachnoid bleed within a sulcus at the right vertex occurred in a patient with 94\% platelet inhibition and PRU 21, who had a right supraclinoid carotid aneurysm treated by placement of a flow-diverting stent. The other patient had a large retroperitoneal hemorrhage, with levels of platelet inhibition measured at $72 \%$ and PRU 83 at the time of the procedure. Because this patient received premedication as a single 450-mg dose of clopidogrel 5 hours before the procedure, this may actually be an underestimation of the achieved platelet inhibition. A large subarachnoid hemorrhage in 1 patient was related to on-table aneurysm perfo- ration, so it was not included in the bleeding group for statistical analysis. Minor bleeding events in 7 (15\%) patients were small groin hematomas of little clinical significance.

Clopidogrel response was greater in the 10 patients with a bleed of any size (median platelet inhibition, 63.5\%; IQR, $36 \%-75 \%$ ) compared with those with no bleeding complication (median platelet inhibition, 19\%; IQR, 11\%-47\%), resulting in a median difference between the degree of platelet inhibition in bleeding and nonbleeding groups of 38\% (95\% CI, $10.6 \%-65.4 \% ; P=.015)$.

Comparison between clopidogrel response in the 3 patients with a major bleed and those with minor or no bleeding demonstrated a more pronounced difference, with median platelet inhibition of $94 \%$ (IQR, 72\%-99\%) versus $24 \%$ (IQR, $11.5 \%$ $55.5 \%$ ), resulting in a median difference between percentage platelet inhibition in these 2 groups of $69 \%$ (95\% CI, 23\%-100\%; $P=.008)$.

ROC analysis was undertaken to identify an optimal threshold to define hyper-response to clopidogrel. Using $72 \%$ or greater platelet inhibition as the criterion, 7 patients $(14.9 \%)$ were defined as clopidogrel hyper-responders; $42.8 \%$ of these patients had a major bleeding complication, while all non-hyper-responders were major bleeding complication-free. If we used a threshold of $\geq 72 \%$ inhibition to define hyper-response, the percentage of platelet inhibition by clopidogrel as a diagnostic tool for clinically significant bleeding events demonstrated high diagnostic capacity ( $\mathrm{ROC}$ area $=0.96 ; 95 \% \mathrm{CI}, 0.89-1)$, resulting in sensitivity of $100 \%$ and specificity of $90.9 \%$. Due to the small sample size and low number of events, caution is required in the extrapolation of these results into clinical practice; larger studies would be required to define and validate a robust threshold for clinical use.

Including minor with major bleeding in the ROC analysis results in a lower diagnostic capacity (ROC area $=0.75 ; 95 \% \mathrm{CI}$, $0.58-0.93$ ), with a threshold of $\geq 53 \%$ inhibition corresponding to a sensitivity of $70 \%$ and a specificity of $75.7 \%$, and is of limited clinical utility. The incidents of minor bleeding in our study were all small groin hematomas, a complication that may be influenced by puncture technique and by achieving inadequate hemostasis at the end of the procedure. Also, because the cutoff below which patients are deemed to be low responders has variably been nominated as anywhere between $23 \%$ and $40 \%$ platelet inhibition, ${ }^{6,7}$ use of this lower threshold would necessarily result in a narrow therapeutic window.

\section{Systemic Anticoagulation with Intravenous Heparin and Bleeding Events}

When divided by bleeding outcome, mean ACT was similar among all groups with 392.2 (95\% CI, 354.32-430.15) in those with no bleeding complication, 373.3 (95\% CI, 315.67-431) in those with any bleed, and 375 (95\% CI, 207.9-542.1) in those with clinically significant bleed. 


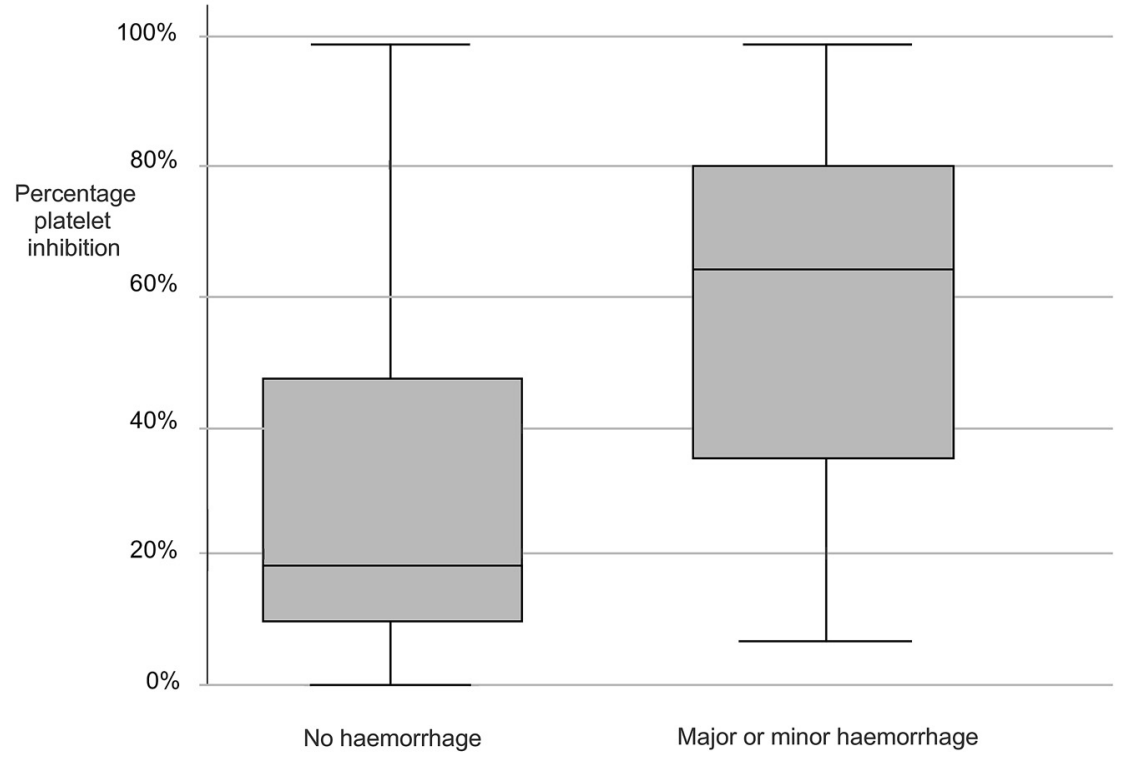

FIG 3. Percentage of platelet inhibition for patients with major or minor hemorrhagic complications, compared with those with no hemorrhagic complications.

In the patients whose intravenous heparin was continued in the ward, the mean aPTT was also similar between each of the bleeding outcomes: the postprocedure aPTT was 63.3 (95\% CI, (51.09-75.57) in those with no bleeding complications, 60.4 (95\% CI, 49.38-71.38) in those with any bleed, and 60.6 (95\% CI, $54.66-66.47)$ in those with a clinically significant bleed.

\section{DISCUSSION}

To our knowledge, no previous studies have investigated clopidogrel hyper-response and bleeding risk in the neurointervention population, though a few studies have examined variation in clopidogrel response in this population without describing how the rate of bleeding complications correlated with platelet function test results. ${ }^{18-21}$

Two previously published studies in the cardiac population that specifically address bleeding complications in the context of antiplatelet medication showed a strong correlation between clopidogrel hyper-response and bleeding risk. Sibbing et $\mathrm{al}^{11}$ found that $38 \%$ of patients undergoing percutaneous coronary intervention were hyper-responders, who had a higher incidence of major bleeding $(2.2 \%)$ compared with the remainder of the population $(0.8 \%)$. Cuisset et $\mathrm{al}^{12}$ assessed bleeding risk in the first month after discharge of patients with acute coronary syndrome on dual antiplatelet therapy, finding that the risk of major or minor bleeding was $6.6 \%$ for those in the first quartile of clopidogrel response compared with $1.4 \%$ in the remainder.

This study showed that a strong correlation between the degree of platelet inhibition by clopidogrel and the incidence of periprocedural bleeding complications also exists in patients undergoing elective neurointerventional procedures.

Systemic anticoagulation with heparin remains a potential confounding factor linking clopidogrel hyper-response with an increased risk of perioperative bleeding in this population. The mean intraoperative ACT for the study population was at the upper end of the acceptable range, which could increase the risk of bleeding events regardless of the individual antiplatelet response to clopidogrel. However, because all patients received a similar dose of intraprocedural heparin and there were no differences in ACT measurements between bleeding and nonbleeding groups, the relatively high intraprocedural anticoagulation achieved does not unduly alter the validity of our hypothesis.

Of potential concern is the influence of postprocedural heparin on our results, given that the 2 intracranial hemorrhages occurred in patients who received intravenous heparin following the procedure. However, when the patients who received heparin infusions were divided into groups based on bleeding outcome, there were no differences in the mean aPTT achieved by each group, in contrast to the large difference in clopidogrel effect between the groups.

While the relatively high intraoperative ACT and the use of heparin anticoagulation in the postprocedural period may well potentiate the effects of antiplatelet medication on bleeding risk, they do not account for the preponderance of bleeding events among the patients with a high level of response to the antiplatelet effects of clopidogrel.

The possibility that the type of intervention performed may impact bleeding risk is another consideration. The 2 small intracranial hemorrhages in this study group occurred in patients who had undergone placement of a flow-diverting stent. However, it is difficult to implicate traction on arteries or altered flow dynamics related to stent placement in the etiology of these events, given that the bleeding site was distant from the treated vessel in both cases. The early experience of this method of aneurysm treatment emphasizes the potential thrombotic complications of parent vessel occlusion, small artery occlusion, and in-stent thrombosis. A multicenter study of 70 patients treated with the Silk flow-diverting stent (Balt Extrusion, Montmorency, France) reported 3 extracranial hemorrhages but no intracranial hemorrhage. ${ }^{22} \mathrm{~A}$ multicenter study of 31 patients treated with the Pipeline flowdiverting device (Covidien, Irvine, California) reported no hemorrhagic complications. ${ }^{23}$

A more likely mechanism by which flow-diverting stents could affect periprocedural bleeding risk is in their requirement for postprocedural anticoagulation with intravenous heparin, which accounts for the high number of such patients in our study. In addition, these patients continue to receive at least 6 months of antiplatelet medication, usually with clopidogrel. While the administration of heparin did not significantly affect the results of this study, the need for ongoing clopidogrel administration highlights the potential for clopidogrel hyper-response to increase the cumulative risk of adverse bleeding events.

Differences between patients undergoing neurointerventional procedures and the cardiac patients who have been studied in greater numbers may influence the clinical impact of interindi- 
vidual variability in the clopidogrel response. In concentrating on elective patients and excluding those with a recent ischemic or hemorrhagic event, our study population is strongly weighted toward patients undergoing treatment for a cerebral aneurysm. These patients tend to be younger with fewer cardiovascular risk factors than those undergoing cerebrovascular or coronary revascularization, so the background risk of thromboembolic events may be reduced. Whether this is offset, or perhaps overshadowed, in the periprocedural period by the need for catheterization of intracranial arteries is uncertain, but regardless, it may have implications for clopidogrel hyper-responders during the 6-12 months postprocedure when patients with intracranial stents will receive maintenance dual antiplatelet therapy. It is possible that a given antiplatelet dose in a neurointervention patient will be associated with an increased bleeding risk compared with patients undergoing cardiac percutaneous coronary intervention or cerebrovascular stent placement.

Authors of future research into the identification and management of clopidogrel hyper-response should bear in mind the lessons from previous research into hypo-response. Despite the large body of evidence available linking low clopidogrel response to an increased risk of adverse cardiovascular outcomes, differences in assay devices and the choice of statistical methods to define "hyporesponse" mean that researchers have struggled to achieve consensus on the optimal cutoff defining low response.

A different approach to understanding the impact of variability in the clopidogrel response relies on identifying genetic polymorphisms in the CYP2C19 allele that are associated with high or low response. The CYP2C19*2 variant is the most common of several loss-of-function alleles. ${ }^{5,24}$ Conversely, the CYP2C19*17 variant allele causes increased metabolism and function of clopidogrel and has been shown to reduce the incidence of major adverse cardiovascular events in patients with acute coronary syndrome. ${ }^{25,26}$

A target of future research would be a prospective study comparing genetic testing with measures of platelet aggregation to determine which is best able to predict the risk of an adverse bleeding outcome. The high specificity of genetic testing may prove to be a drawback, given the multifactorial etiology of clopidogrel response variability, and the time required to perform genetic testing may reduce its clinical utility in comparison with point-of-care assay devices. In addition, genetic testing will need to include the full panel of CYP2C19 alleles because there is evidence that the combination of high- and low-function variants will result in a low-to-normal function phenotype. ${ }^{27}$

\section{CONCLUSIONS}

Hyper-response to the antiplatelet effects of clopidogrel is associated with a significant increase in bleeding risk in the setting of endovascular neurointervention. Any proposed threshold value for a clinically significant increased risk of major bleeding will require validation with a larger study population. Testing for genetic polymorphisms associated with increased platelet inhibition by clopidogrel is a promising avenue of further research. However, point-of-care assay devices retain the advantage of speed and simplicity of use and will include the effects of nongenetic determinants of platelet function. Standardization of study methodol- ogy in future research protocols is of critical importance to provide statistically robust and clinically useful thresholds defining high and low response to clopidogrel.

Disclosures: Peter Mitchell—UNRELATED: Travel/Accommodations/Meeting Expenses Unrelated to Activities Listed: ev3, Comments: Neuro Exchange scientific meeting attendance, Other: Boston Scientific, Comments: unrestricted clinical research nurse support.

\section{REFERENCES}

1. Bhatt DL, Kapadia SR, Bajzer CT, et al. Dual antiplatelet therapy with clopidogrel and aspirin after carotid artery stenting. I Invasive Cardiol 2001;13:767-71

2. Cohen M. Antiplatelet therapy in percutaneous coronary intervention: a critical review of the 2007 AHA/ACC/SCAI guidelines and beyond. Catheter Cardiovasc Interv 2009;74:579-97

3. Snoep JD, Hovens MM, Eikenboom JC, et al. Clopidogrel nonresponsiveness in patients undergoing percutaneous coronary intervention with stenting: a systematic review and meta-analysis. $A m$ Heart J 2007;154:221-31

4. Musallam KM, Charafeddine K, Bitar A, et al. Resistance to aspirin and clopidogrel therapy. Int J Lab Hematol 2011:33:1-18

5. Scott SA, Sangkuhl K, Gardner EE, et al. Clinical Pharmacogenetics Implementation Consortium Guidelines for Cytochrome P4502C19 (CYP2C19) Genotype and Clopidogrel Therapy. Clin Pharmacol Ther 2011;90:328-32

6. Ari H, Ozkan H, Karacinar A, et al. The EFFect of hIgh-dose ClopIdogrel treatmENT in patients with clopidogrel resistance (The EFFICIENT Trial). Int J Cardiol 2012;157:374-80

7. Campo G, Fileti L, de Cesare N, et al. Long-term clinical outcome based on aspirin and clopidogrel responsiveness status after elective percutaneous coronary intervention. J Am Coll Cardiol 2010;56: 1447-55

8. Price MJ, Endemann S, Gollapudi RR, et al. Prognostic significance of post-clopidogrel platelet reactivity assessed by a point-of-care assay on thrombotic events after drug-eluting stent implantation. Eur Heart J 2008;29:992-1000

9. Aradi D, Komócsi A, Vorobcsuk A, et al. Prognostic significance of high on-clopidogrel platelet reactivity after percutaneous coronary intervention: systematic review and meta-analysis. Am Heart J 2010;160:543-51

10. Holmes DR, Dehmer GJ, Kaul S, et al. ACCF/AHA clopidogrel clinical alert: approaches to the FDA "boxed warning"-a report of the American College of Cardiology Foundation Task Force on Clinical Expert Consensus Documents and the American Heart Association. Circulation 2010;122:537-57

11. Sibbing D, Schultz S, Braun S, et al. Antiplatelet effects of clopidogrel and bleeding in patients undergoing coronary stent placement. J Thromb Haemost 2010;8:250-56

12. Cuisset T, Cayla G, Frere C, et al. Predictive value of post-treatment platelet reactivity for occurrence of post-discharge bleeding after non-ST elevation acute coronary syndrome: shifting from antiplatelet resistance to bleeding risk assessment? Eurointervention 2009;5:325-29

13. Serebruany V, Rao SV, Silva MA, et al. Correlation of inhibition of platelet aggregation after clopidogrel with post discharge bleeding events: assessment by different bleeding classifications. Eur Heart J 2010;31:227-35

14. van Werkum JW, Harmsze AM, Elsenberg EH, et al. The use of the VerifyNow system to monitor antiplatelet therapy: a review of the current evidence. Platelets 2008;19:479-88

15. Varenhorst C, James S, Erlinge D, et al. Assessment of P2Y(12) inhibition with the point-of-care device VerifyNow P2Y12 in patients treated with prasugrel or clopidogrel coadministered with aspirin. Am Heart J 2009; 157:562.e1-e9

16. Cuisset T, Frere C, Poyet R, et al. Clopidogrel response: head-tohead comparison of different platelet assays to identify clopidogrel

AJNR Am J Neuroradiol 34:721-26 Apr 2013 www.ajnr.org

725 
non responder patients after coronary stenting. Arch Cardiovasc Dis 2010;103:39-45

17. Breet NJ, van Werkum JW, Bouman HJ, et al. Comparison of platelet function tests in predicting clinical outcome in patients undergoing coronary stent implantation. JAMA 2010;303:754-62

18. Ryu DS, Hong C-K, Sim YS, et al. Anti-platelet drug resistance in the prediction of thromboembolic complications after neurointervention. J Korean Neurosurg Soc 2010;48:319-24

19. Müller-Schunk S, Linn J, Peters N, et al. Monitoring of clopidogrelrelated platelet inhibition: correlation of nonresponse with clinical outcome in supra-aortic stenting. AJNR Am J Neuroradiol 2008;29: 786-91

20. Prabhakaran S, Wells KR, Lee VH, et al. Prevalence and risk factors for aspirin and clopidogrel resistance in cerebrovascular stenting. AJNR Am J Neuroradiol 2008;29:281-85

21. Lee $\mathrm{DH}$, Arat A, Morsi $\mathrm{H}$, et al. Dual antiplatelet therapy monitoring for neurointerventional procedures using a point-of-care platelet function test: a single-center experience. AJNR Am J Neuroradiol 2008;29:1389-94

22. Byrne JV, Beltechi R, Yarnold JA, et al. Early experience in the treat- ment of intra-cranial aneurysms by endovascular flow diversion: a multicentre prospective study. PLoS One 2010;5. pii: e12492

23. Nelson PK, Lylyk P, Szikora I, et al. The Pipeline embolization device for the intracranial treatment of aneurysms trial. AJNR Am J Neuroradiol 2011;32:34-40

24. Bauer T, Bouman HJ, van Werkum JW, et al. Impact of CYP2C19 variant genotypes on clinical efficacy of antiplatelet treatment with clopidogrel: systematic review and meta-analysis. BMJ 2011:343: $\mathrm{d} 4588$

25. Tiroch KA, Sibbing D, Koch W, et al. Protective effect of the CYP2C19 17 polymorphism with increased activation of clopidogrel on cardiovascular events. Am Heart J 2010;160:506-12

26. Sibbing D, Koch W, Gebhard D, et al. Cytochrome $2 \mathrm{C} 19^{\star} 17$ allelic variant, platelet aggregation, bleeding events, and stent thrombosis in clopidogrel-treated patients with coronary stent placement. Circulation 2010;121:512-18

27. Sibbing D, Gebhard D, Koch W, et al. Isolated and interactive impact of common CYP2C19 genetic variants on the antiplatelet effect of chronic clopidogrel therapy. J Thromb Haemost 2010;8: 1685-93 